-. $\quad \sqrt{ }$ May 1980

BNL -27985

CONF-800213--12

Present Status of the VMI and Related Models

Gertrude Scharff-Goldhaber

Cornel1 University and Brookhaven National Laboratory

MASTER

Upton, New York 11973

\author{
Invited Paper \\ International Conference on Band Structure and Nuclear Dynamics \\ New Orleans, Louisiana \\ February 28 - March 1, 1980
}

\begin{tabular}{l} 
This book was prepared as an account of work sponsored by an agency of the United States Government. \\
Neither the United States Government nor any agency thereof, nor any of their employees, makes any \\
warranty, express or implied, or assumes any legal liability or responsibility for the accuracy. \\
completeness, or usefulness of any information, apparatus, product, or process disccosed, or \\
represents that its use would not infringe privately orwned rights. Reference herein to any specific \\
commercial product, process, or service by trade name, trademark, manufacturer, or otherwise, does \\
not necessarily constitute or imply its endorsement, recommendation, or favoring ty the United \\
States Government ur any agency thereof. The views and opinions of authors expressed herein do not \\
necessarily state or reflect those of the United States Government or any agency thereof. \\
\hline
\end{tabular}

The submitted manuscript has been authored under contract DE-AC02-76CH00016 with the U.S. Department of Energy. Accordingly, the U.S. Government retains a nonexclusive, royalty-free license to publish or reproduce the published form of this contribution, or allow others to do so, for U.S. Government purposes. 


\section{DISCLAIMER}

This report was prepared as an account of work sponsored by an agency of the United States Government. Neither the United States Government nor any agency Thereof, nor any of their employees, makes any warranty, express or implied, or assumes any legal liability or responsibility for the accuracy, completeness, or usefulness of any information, apparatus, product, or process disclosed, or represents that its use would not infringe privately owned rights. Reference herein to any specific commercial product, process, or service by trade name, trademark, manufacturer, or otherwise does not necessarily constitute or imply its endorsement, recommendation, or favoring by the United States Government or any agency thereof. The views and opinions of authors expressed herein do not necessarily state or reflect those of the United States Government or any agency thereof. 


\section{DISCLAIMER}

Portions of this document may be illegible in electronic image products. Images are produced from the best available original document. 


\title{
PRESENT STATUS OF THE VMI AND RELATED MODELS*
}

Gertrude Scharff-Goldhaber

Corne11 University and Brookhaven National Laboratory

Upton, New York 11973

USA

\begin{abstract}
This article traces the evolution of the Variable Moment of Inertia model in its relation to the shell model, the Bohr-Mottelson model and the Interacting Boson Model. The discovery of a new type of spectrum, that of "pseudomagic" nuclei (isobars of doubly magic nuclei) is reported, and an explanation for their dynamics is suggested. The type of rotational motion underlying the ground state band of an e-e nucleus is shown to depend on whether the minimum number of valence nucleon pairs of one kind (neutrons or protons) is $<2$ or $>2$. In the former case the "alphadumbbell model" holds; in the latter the "two-fluid model."
\end{abstract}

In the preceding talk Abe Klein, who has in the past contributed significantly to our field, discussed the relationship of the mathematical expressions corresponding to various nuclear models. I shall in the following present a phenomenological account of the Variable Moment of Inertia (VMI) model. As you know, this model has not only been successful in describing spectra of ground state bands $(0+, 2+, 4+, 6+, \ldots)$ in non-magic e-e nuclei, but also those of higher bands, including "yrast" bands above band crossing, and finally, bands in odd-A nuclei ${ }^{1}$ ). A review entitled "The Variable Moment of Inertia Model and Theories of Nuclear Collective Motion"2) gives a detailed account of the manifold aspects of the model, in particular of its relation to shell structure, of modifications proposed by various authors, and of its relation to "microscopic" (HFB) nuclear theory. In this talk I shall concentrate mainly on the VMI model in its two parameter version as it applies to spectra in e-e nuclei before band-crossing takes place, and on the understanding of nuclear dynamics it has yielded.

First, I shall trace the origin of the VMI model and describe its properties. Second, I shall discuss a recently discovered phenomenon, namely the level structure of pseudomagic nuclei. Third, it will be shown that this model permits a general and amazingly precise interpretation of the dynamics of yrast bands in terms of rotational motion based on two distinct nuclear configurations. Fourth, I plan to make a few remarks concerning the resemblance of the "VMI equation of state" for the effective moment of inertia with equations of state of systems of condensed matter, and discuss the significance of this resemblance.

1). The first clue suggesting that spectra of ground state bands in even-even nuclei reflect one and the same mechanism was Edoardo Mallmann's ${ }^{3}$ ) observation (1959) that the energy ratios $\mathrm{R}_{\mathrm{J}} \equiv \mathrm{E}_{\mathrm{J}} / \mathrm{E}_{2}$ for $\mathrm{J}=6$ and 8 lie on "universal" curves if plotted vs. $R_{4}$. He concluded on the basis of rather meager data that the universal curves range over $1 \leq R_{4} \leq 3.33$ (the rotational limit). This finding implied that the spectrum $E_{J}$ can be presented as a function of two parameters characteristic for a given nucleus. With the aid of Arthur Kerman, Mallmann searched for a constraint of a triaxial nucleus model which might yield the "Mallmann curves," but their attempt failed. 
Mallmann's startling phenomenological result was almost totally ignored by theorists. The reasons for this neglect are somewhat complex. They may perhaps be most easily understood from fig. 1 which depicts the chronology of events that took place after the introduction and general acceptance of the nuclear shell model in the late forties: To the right of this figure one finds the well-known level scheme of the Bohr-Mottelson strongly deformed nucleus, whose level energies are inversely proportional to the effective moment of inertia, which was thought to be independent of the state $\mathrm{J}$. In order to take rotation-vibration

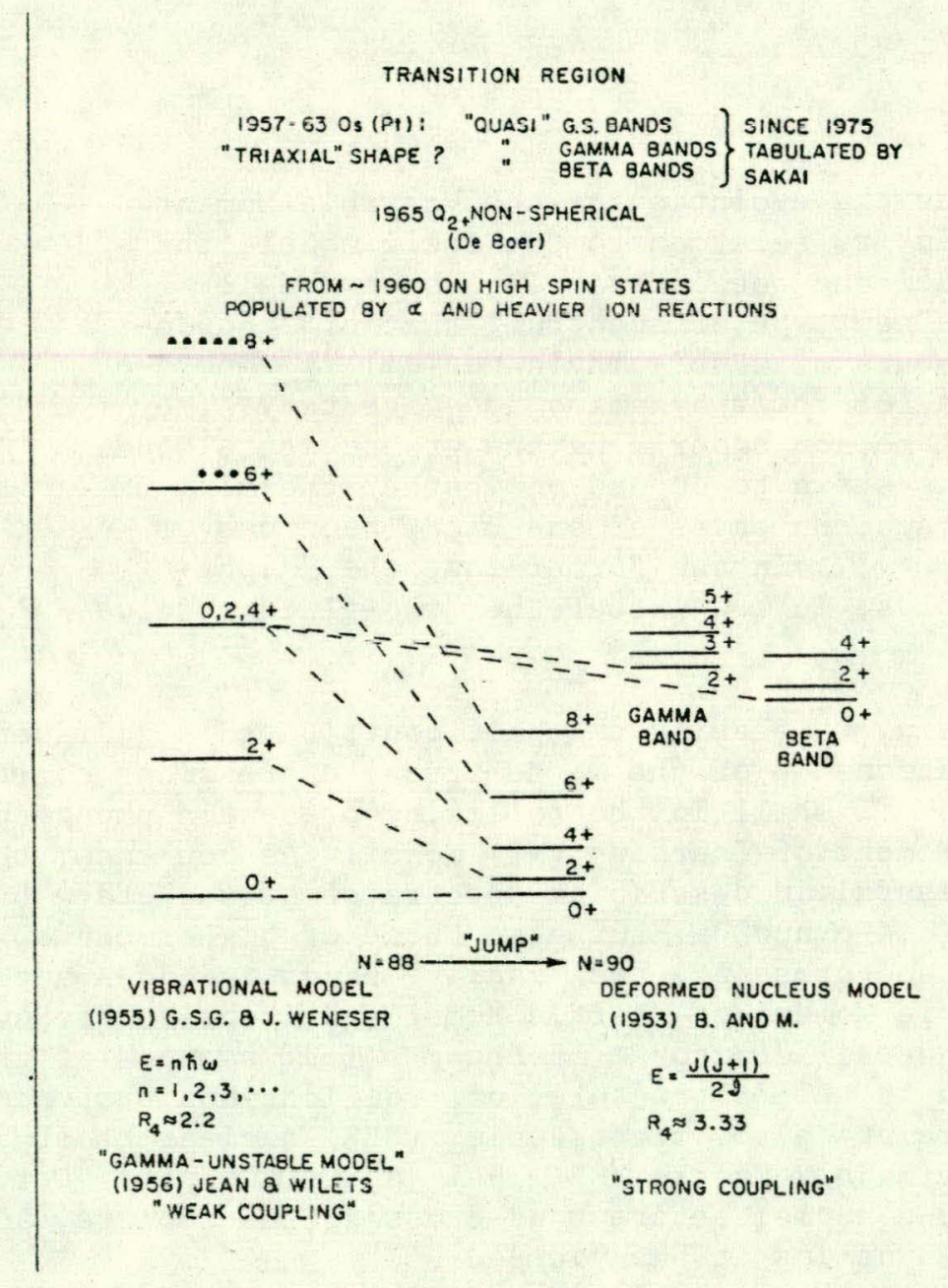

Fig. 1. Schematic graph depicting evolution of models of collective motions in e-e nuclei from the early 1950s on.

mixing (as observed in molecules) into account, a second term $\propto[\mathrm{J}(\mathrm{J}+1)]^{2}$ with a very small coefficient was thought to suffice. Surprisingly, the resulting value for $S$ was found to be 2 to 3 times smaller than that of a rigid rotor; this posed a difficulty which Bohr and Mottelson first tried to resolve by introducing the idea of "irrotational" motion. However, the resulting irrotational $\&$ turned out to be 4 ot 5 times smaller than the observed moment of inertia. The region or regions in the chart of nuclides where rotational bands occur, i.e. the regions with many valence nucleons, were thought to be governed by "strong coupling." A simultaneous study of all known excited states in even-even nuclei which I carried out showed, however, that only if both numerous neutron and proton pairs are present, low-1ying $2+$ states as observed in rotational bands ( $40 \rightarrow 130 \mathrm{keV})$ occur. It soon became clear that in regions with fewer valence nucleons a different but also uniform type of band structure obtains, namely a "near-harmonic" structure for which the second excited state (or cluster of states) has $\sim 2.2$ times the energy of the first $2+$ state. This, together with the previously reported "Kraushaar- 
Goldhaber" branching ratios for transition rates occurring in this type of level scheme encouraged J. Weneser and myself to propose a "vibrational model," which was the antecedent of the interacting boson (or phonon) models. This model had remarkable predictive powers, both with regard to level energies and transition probabilities. Bohr and Mottelson's new theory of nuclear collective motions for the domain of weak coupling (i.e. few valence nucleons) offered a basis for such a model, in which the bosons are provided by $n \hbar \omega(n=1,2,3, \ldots)$ quadrupole oscillations of the surface of the closed shell core, to which a few $\left(\mathrm{e} . \mathrm{g} \cdot \mathrm{f}_{7 / 2}^{4}\right)$ valence nucleons are weakly coupled, thus lifting the degeneracy of the multiplets. However, I was troubled right from the start by the fact that not only are the energies of the first $2+$ states in singly or doubly closed shell nuclei excessively high, but also the energy ratios of levels are far from near-harmonic: they range from $\sim 1.8>E_{2} / E_{1}>1$ (where $E_{1}$ and $E_{2}$ denote the first and second excited states $)^{4}$ ). Very soon afterwards, Wilets and Jean based their "gamma-unstable" model on the experimental evidence we had presented. As we now know, the symmetry properties of the "vibrational" model are in harmony with Iachello and Arima's SU(5) group, examples of which occur at the beginning of the rare earths region (i.e. near the beginning of the neutron (82-126) and proton (50-82) shells), whereas the gamma-unstable model is most closely related to the $0(6)$ group which happens to agree best with the level scheme of ${ }^{196} \mathrm{Pt}$ (i.e. close to the end of the shells). But I am getting ahead of myself: As we then pointed out, there is an abrupt jump from the near-harmonic to the rotational structure, as the neutron number increases from 88 to 90 . The question came up whether nuclei can only be either "vibrational" or "rotational" or whether there is an intermediate type of dynamic behavior. The theorist's answer was that this question could only be tackled by intermediate coupling calculations, which were "impossibly messy." Therefore, an experimental approach to this question seemed to be called for. Fortunately, the first 2+ states of osmium nuclei seemed to imply exactly an intermediate behavior, and the very first experimental evidence of band structure found in these nuclei ${ }^{5}$ ) indicated a gradual transition from one type to the other, as shown by the dashed lines in fig. 1. The transition was even more impressive, when energy ratios $E_{J} / E_{2}$ were plotted. This then gave Mallmann the idea to go one step further and plot $R_{J}$ vs. $R_{4}$.

The level schemes of e-e osmium nuclei could be fairly well interpreted in terms of the triaxial model, and even better by a triaxial nucleus which undergoes betavibrations ${ }^{6}$ ). However, interest in these fascinating regularities of nuclear structure was from the middle fifties on somewhat overshadowed by two basic problems, namely that of the moment of inertia (2 to 3 times smaller than rigid) and that of the "gap" (between ground states and first excited states of e-e nuclei at and near closed shells), which were eventually solved with the introduction of a pairing forced followed by the adoption of the BCS method of taking pairing correlations between pairs of "time reversed" nucleons into account (with which the problem of the superconductor had been solved). This development led to interesting calculations of level schemes by Kisslinger and Sorensen and of a few moments of inertia of strongly deformed nuclei (which could be calculated with $20 \%$ accuracy by Griffin and Rich, and by Nilsson and Pryor.) It probably was for this reason that so little attention was paid to Mallmann's brilliant insight. Within the next several years, however, important new results were obtained which stimulated further thought: Gregory Breit's suggestion for a measurement of electric quadrupole moments of $2+$ states $\left(Q_{2+}\right)$ by means of the "reorientation effect" led to the discovery that these states in "vibrational" nuclei have much larger $Q_{2+}$ values than can be accounted for by our original model. At about the same time, a large number of higher spin states were populated in strongly deformed even-even nuclei by means of alpha (or heavier ion) induced reactions. This method (in contrast to the previous experiments carried out exclusively with radioactive nuclei) permitted the study of grossly neutron deficient isotopes. Their level schemes were found to deviate appreciably from the $J(J+1)$ rule. An analysis by $S$. M. Harris in terms of a "fourth order cranking model," which leads to an expansion of the moment of inertia in even terms of $\omega$, the rotational velocity (where the semiclassical relationship $(\xi \omega)^{2}=J(J+1)$ holds), however, allowed very good fits. 
Another approach to the same problem was taken by Diamond and Stephens, who had first fitted such bands with the triaxial model plus beta vibrations, and then discovered, in collaboration with Swiatecki, that an equivalent interpretation of the spectrum is obtained by means of a "beta-stretching model," in which the deformation, just as the interatomic distance in diatomic molecules, is taken to increase with angular momentum. This latter approach, just as Harris', leads to a two parameter expression for the energy levels, but it requires in addition an assumption for the dependence of $\mathcal{S}$ on the deformation $\beta$, i.e. a "hidden" third parameter.

While both these papers were concerned with strongly deformed though unstable nuclei, I felt encouraged by the Mallmann rule to search for an analytical description for the ground state bands of all even-even nuclei. I was joined in this effort by an experimentalist, Mario Mariscotti, who had just completed his Ph.D. thesis in Argentina and came recommended by Mallmann, and later by an English theorist, Brian Buck ${ }^{7}$ ). We started out with the beta stretching model, but found that it was preferable to eliminate the deformation parameter $\beta$ and to determine $\checkmark$ by minimizing $E$ with respect to $\mathscr{S}$, rather than to $\beta$. Hence we named the new model "Variable Moment of Inertia (VMI)" model. Subsequent comparison with Harris' model showed that the two, in their two parameter versions, are completely equivalent.

The dependence of the parameter $s_{0}$ ( $f i g .2$ ) deduced from the observed band energies suggested that the extension of the model to negative values of $\mathfrak{J}_{0} \mathrm{might}$ be possible. Indeed it was found ${ }^{8}$ ) that extension to $\$_{0} \rightarrow-\infty$ results in good agreement with the data ( $f i g .3$ ). The VMI equations, which include a "harmonic" potential energy term, in addition to the rotational term

$$
\begin{aligned}
& E=\frac{C}{2}\left(I-S_{0}\right)^{2}+\frac{J(J+1)}{2 C} \\
& \left.\frac{\partial E}{\partial \mathscr{S}}\right|_{J}=0
\end{aligned}
$$

lead $\operatorname{tog}^{3}-\mathfrak{S}_{0} \mathfrak{g}^{2}=\frac{\mathrm{J}(\mathrm{J}+1)}{2 \mathrm{C}}$

as shown in $\mathrm{fig}$. 4 , both for positive and negative values of $\oint_{0}$. The important point to be kept in mind is that for negative values of $\mathfrak{S}_{0}$ the ground state is spherical: $S(0)=0$, as may be deduced from the left side of fig. 4. Fig. 5 shows the parameters $C$ and $\mathcal{G}_{0}$ in combination with the "scale factor" $E_{2}$, as well as the hardness parameter $h$, as functions of the measured ratio $R_{4}$, over the whole range of validity of the model. Of particular interest is the relatively minor decrease of $\oint_{0} E_{2}$ starting from the rotational limit $\left(R_{4}=3.33 \ldots\right)$ at which point $\mathcal{S}_{0} E_{2}=3$, almost down to the value $R_{4}=2.23$, at which $\mathcal{S}_{0}$ vanishes, and $\mathrm{E}_{\mathrm{J}} \propto(\mathrm{J}(\mathrm{J}+1))^{2 / 3}$ and $\mathcal{G}_{\mathrm{J}} \propto(\mathrm{J}(\mathrm{J}+1))^{1 / 3}$. The limit of validity of the model occurs at $R_{4}=1.82$, where $E_{J} \propto \sqrt{J(J+1)}$ and $\mathcal{S}_{J} \propto \sqrt{J(J+1)}$. As is apparent from $f i g .3$, below $\mathrm{R}_{4}=1.82$ only bands for singly and doubly magic nuclei occur (with one exception, which will be discussed in section 2). The interpretation of this behavior is quite obvious: The higher the "threshold energy" $\frac{C}{2} S_{0}{ }^{2}$, the more does the nucleus resist cranking. As the magic number is reached (i.e. when all valence nucleon pairs have been removed) at $R_{4}=1.82$, the nucleus, now being perfectly spherical, can only be excited by promoting one or two nucleon pairs to a higher orbit, thus bringing about deformation, which permits cranking from the $2+$ state on.

Das, Dreizler and $\mathrm{Klein}{ }^{9}$ ) presented a proof showing that the VMI and Harris models are equivalent to all orders. They also compared the VMI model which describes "yrast bands" in terms of rotational motions with an anharmonic vibrator model, which yields a description of these bands in terms of the empirical "Ejiri formula" : 
GROUND STATE MOMENTS OF INERTIA OF EVEN-EVEN NUCLEI

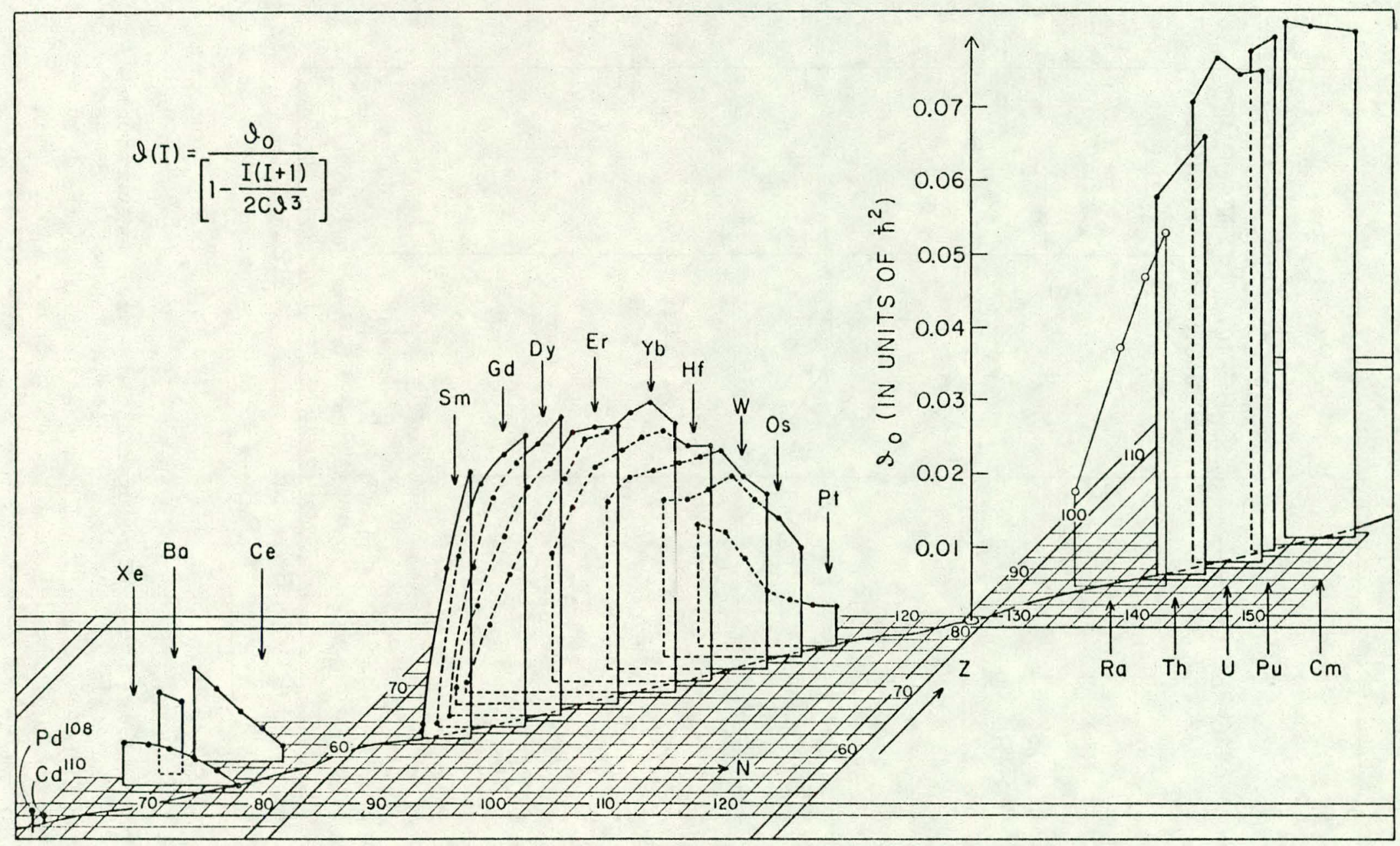

Fig. 2. Three dimensional diagram of $\mathscr{A}_{c}$ (computed from eqs. 1 and 3 ) vs. $N$ and $Z$. (This figure was first published in ref. 7.) 


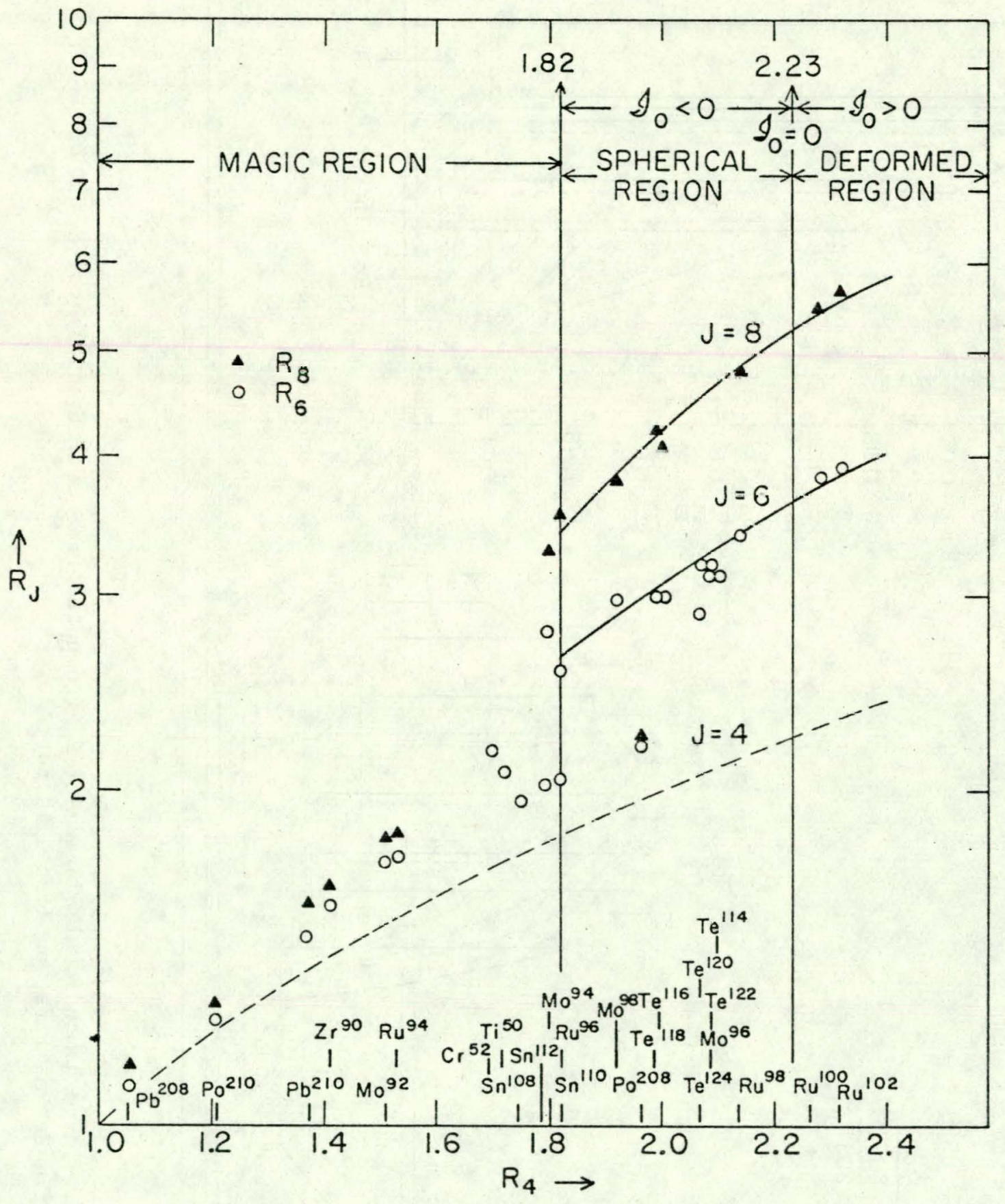

Fig. 3. Ratios $E_{6} / E_{2}$ and $E_{8} / E_{2}$ (logarithmic scale) as functions of $E_{4} / E_{2}$ for the magic and spherical regions. The solid curves are computed from eqs. 1 and 3 . The dashed line indicates $R_{4}$. Only the beginning of the deformed region is shown. In the magic zone the $R_{4}, R_{6}$, and $R_{8}$ values are almost degenerate. In ${ }^{208}$ Po $\left(R_{4}=1.96\right)$, "backbending" occurs already above the $4+$ state. This figure was reproduced from ref. 8. 

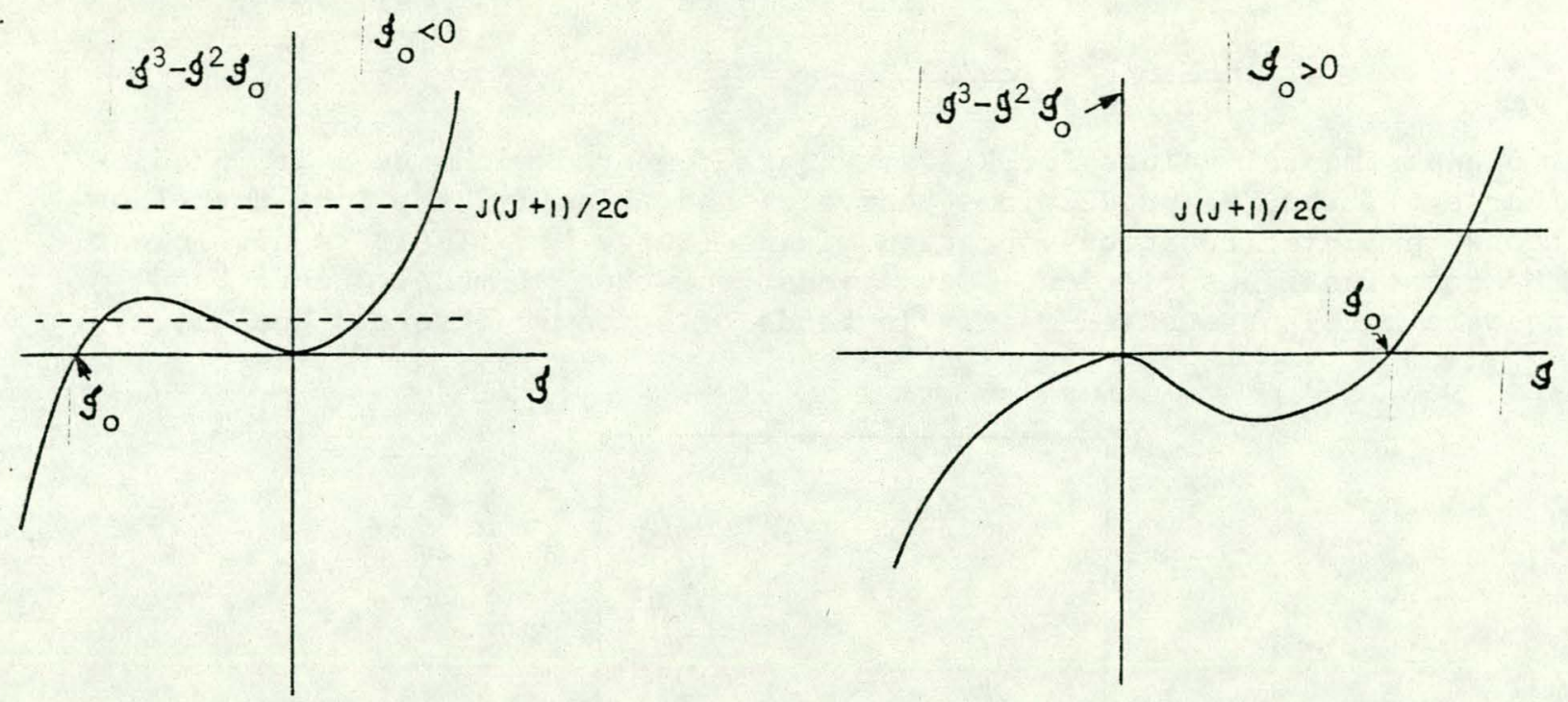

Fig. 4. Graphic solution of eq. 3 for positive (right) and negative (left) values of $\mathcal{S}_{0}$. Only positive values of $\mathcal{f}$ are physically meaningful; for $s_{0}<0$ one obtains $\mathfrak{S}(0)=0$.

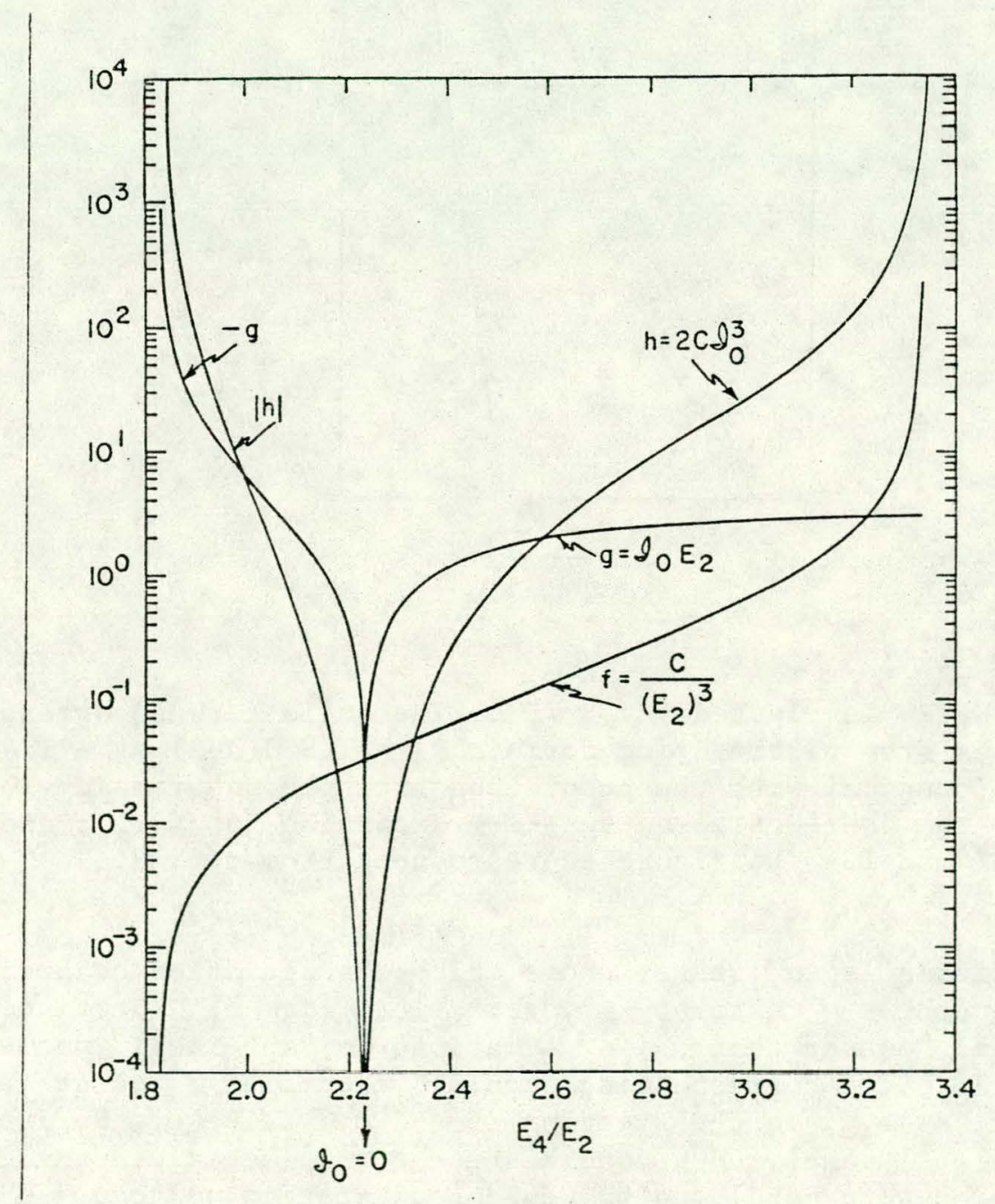

Fig. 5. The functions $f=C /\left(E_{2}\right)^{3}, g=\mathfrak{s}_{0} E_{2}$, and $\left.h=\mid 2 C\right\}_{0}^{3} \mid$ are plotted vs. $E_{4} / E_{2}$. The physical interpretations of singularities occurring at $E_{4} / E_{2}=1.82$ and 2.23 are discussed in sections 1 and 4 . 


$$
\begin{aligned}
& E_{J}=a J+k J(J+1) \text {, which leads to } \\
& R_{J}=\frac{J(J-2)}{8} R_{4}-\frac{J(J-4)}{4} .
\end{aligned}
$$

In $\mathrm{fig} .6$ experimental values for $R_{8}$ vs. $R_{4}$ are compared with the Ejiri predictions (curve a), the VMI predictions (curve c) and, finally, the Bohr-Mottelson predictions including rotation-vibration mixing (curve b). Their results clearly favor the rotational description. Deviations from the VMI model due to band crossing were at first studied mainly in bands of strongly deformed nuclei, e.g.

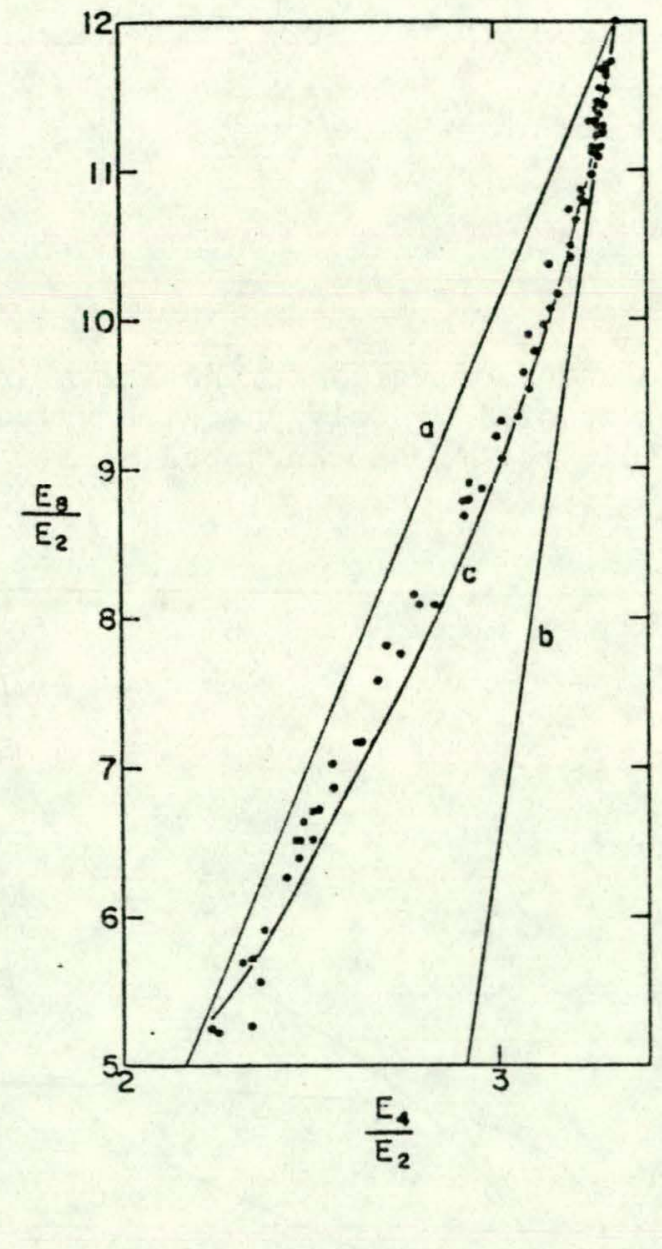

Fig. 6. $E_{8} / E_{2}$ is plotted vs. $E_{4} / E_{2}$. The solid curves correspond to predictions from a) the Ejiri formula $R_{J}=1 / 8 \mathrm{~J}(\mathrm{~J}-2) R_{4}-1 / 4 \mathrm{~J}(\mathrm{~J}-4)$, which is identical with the prediction from the anharmonic vibrator model; b) the Bohr-Mottelson two-term expansion in $\mathrm{J}(\mathrm{J}+1)$; and c) the VMI model. The figure is reproduced from ref. 9.

in ${ }^{158} \mathrm{Dy}$, whose "critical spin" state, above which the deviation occurs, is $\mathrm{J}_{\mathrm{Cr}}=12$, as seen in the now customary $\mathcal{f}$ vs. $\omega^{2}$ plot (fig. 7). Very soon afterwards it became clear however that while deviations in "spherical" nuclei occur at considerably lower $\mathrm{J}_{\mathrm{cr}}$, the corresponding angular velocities $\omega$ are at least as high or even higher, as e.g. in the spectrum of $104 \mathrm{Pd}$. L. Peker, S. Pearlstein and $\mathrm{J}$. Hamilton have recently analyzed ground state bands in actinides, namely ${ }^{232} \mathrm{Th}$, ${ }^{238} \mathrm{U}$, and ${ }^{248} \mathrm{Cm}$, which were populated by Coulomb excitation up to $\mathrm{J}=28$ at GSI in Darmstadt. No band crossing was observed, which may be attributed to the fact that these nuclei have very low rotational energies (and hence angular velocities). It is interesting to note that although VMI model fits with more than two parameters improved agreement with the data, as might be expected, even with just two parameters fits within $1 \%$ of the observed level energies were obtained. 


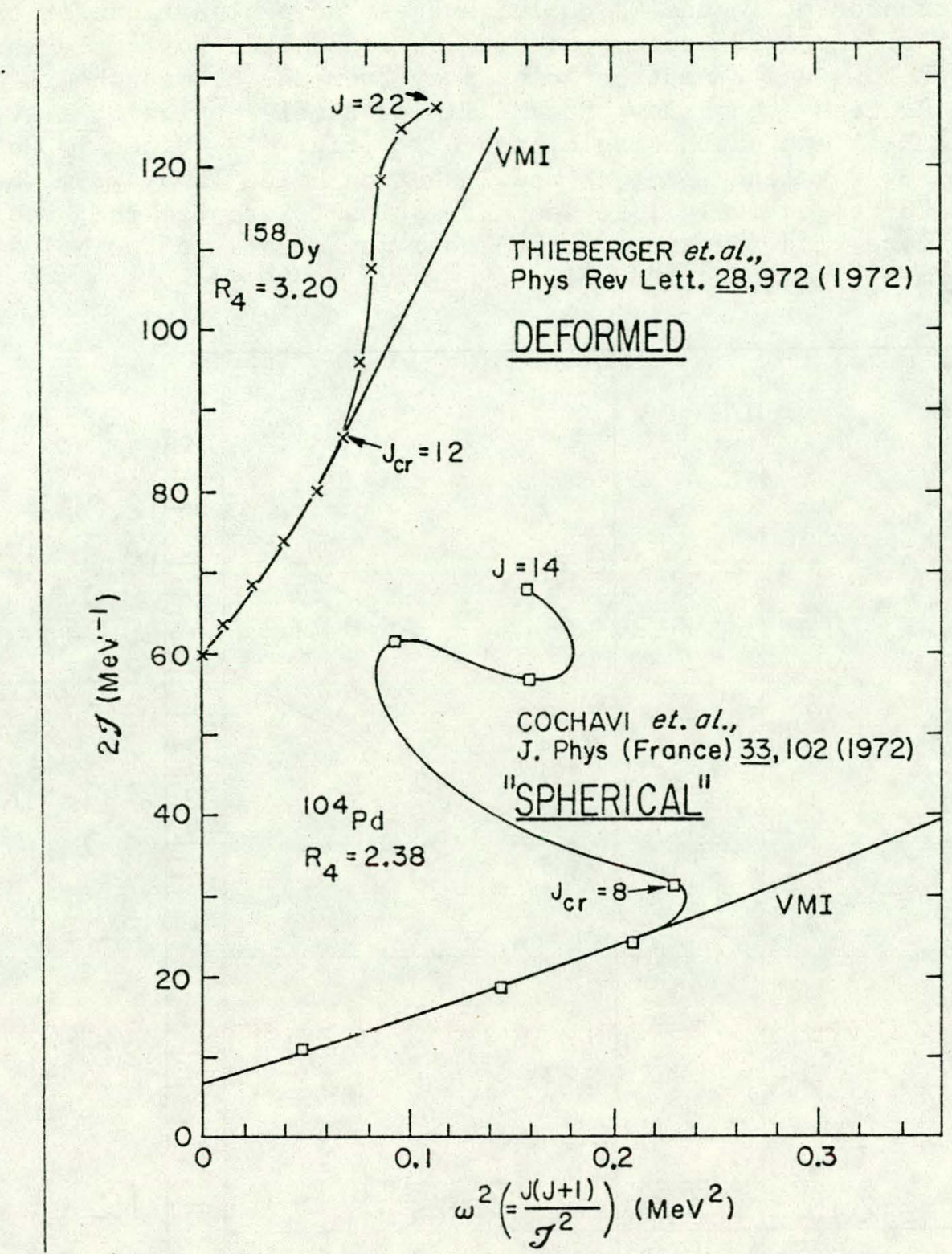

Fig. 7. Comparison between an $\Im$ vs. $\omega^{2}$ plot for a neutron deficient rotor (158 Dy) $\left(E_{2}=98.94 \mathrm{keV} ; C=2.64\left(10^{6} \mathrm{keV}^{3}\right)\right)$ with that of a "vibrator" (104 $\mathrm{Pd})\left(E_{2}=555.8 \mathrm{keV} ; \mathrm{C}=10.23\left(10^{6} \mathrm{keV}^{3}\right)\right)$. The VMI predictions are shown by solid lines. For the rotor $\mathrm{J}_{\mathrm{c}}=12$, while for the vibrator $J_{c}=6$ or 8 .

2). The recognition of the existence of "pseudomagic nuclei"10) evolved from a new, more stringent test of the validity of the Variable Moment of Inertia (VMI) equations near the lower limit, i.e. for near-magic nuclei. Whereas previously only states with $\mathrm{J} \leq 8$ had been known in this region, the present test includes states with $\mathrm{J} \leq 14$. As stated before, between $\mathcal{S}_{0}=0$ and $\mathcal{S}_{0}=-\infty$, the ground state moment of inertia is $G(0)=0$, and nuclei in this region are one or two pairs of particles or holes away from singly magic ${ }^{8}$ ). Below $R_{4}=1.82$ are found yrast bands of singly and doubly magic nuclei, which are characterized by appreciably higher excitation energies of the first $2+$ state than their non-magic neighbors.

At the time the extended VMI mode $1^{8}$ ) was proposed, the only striking exception noted was for ${ }^{208} \mathrm{Po}$ which, with $\mathrm{R}_{4}=1.96$, lies in the "spherical region"

$\left(1.82 \leq \mathrm{R}_{4} \leq 2.23\right.$ ) (fig. 3). We stated then that one is tempted to include ${ }^{208} \mathrm{Po}$ in the "magic branch," which extends to the right from $R_{4}>1$. The results of a 
study ${ }^{11}$ ) of the yrast bands of Te and Cd nuclei suggest an explanation for the exceptional structure of the ${ }^{208}$ Po spectrum: it was found that for ${ }^{132} \mathrm{Te}$ which possesses 2 valence protons and 2 neutron holes away from the closed shells 50 and 82 respectively, $R_{4}$ lies even below the VMI limit, namely at 1.716 , i.e. in the magic region, and $\mathrm{R}_{6}$ lies "on the magic branch"! (fig. 8). Since ${ }_{84}^{208 \mathrm{Po}_{124}}$ is also characterized by 2 valence protons and 2 neutron holes (away from the magic number 82 and 126 respectively), it seems tempting to propose the hypothesis that two particles of one kind (neutrons or protons) may couple to two holes of the other kind to form a "pseudomagic" nucleus.

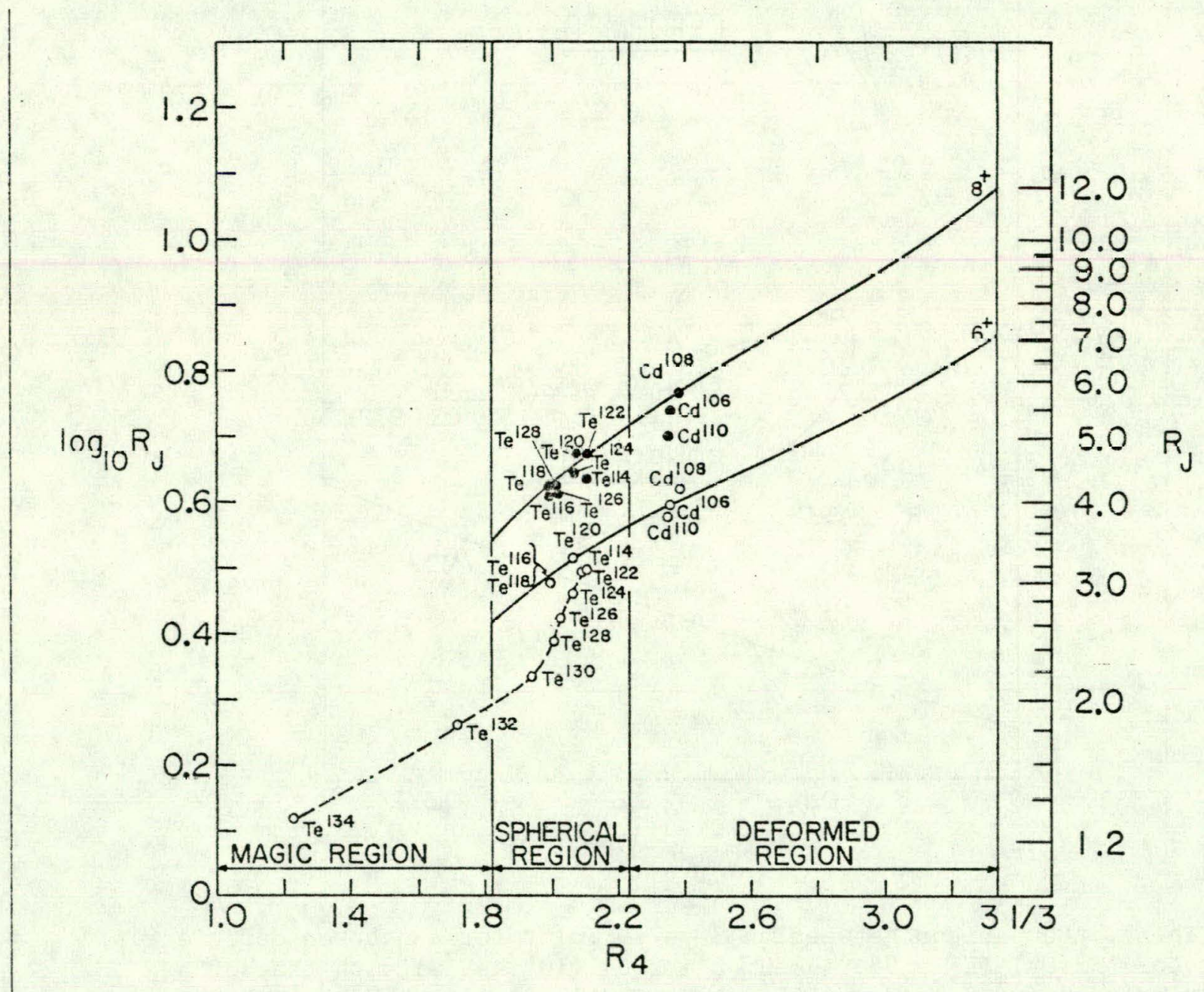

Fig. 8. Log plot of $R_{J}$ vs. $R_{4}$. The $R_{J}$ values for the neutron rich Te nuclei deviate increasingly from the model predictions as $\mathrm{N}=82$ is approached, i.e. "backbending" occurs already above the $4+$ state.

A previously started experimental investigation of nuclei pertaining to the "spherical" region, $1.82 \leq \mathrm{R}_{4} \leq 2.23$, namely of the neutron deficient $\mathrm{Pd}(\mathrm{Z}=46)$ nuclei, appears to support this hypothesis. Whereas in ref. ${ }^{8}$ ) only states with $\mathrm{J} \leq 8$ were included, we populated yrast bands in ${ }^{104} \mathrm{Pd}^{12}$ ), ${ }^{102} \mathrm{Pd}$ and ${ }^{100} \mathrm{Pd}^{13}, 14$ ), and $\left.{ }^{98} \mathrm{Pd} 15\right)$, up to $\mathrm{J}=14$. While the results for ${ }^{98} \mathrm{Pd}{ }^{15}$ ) and $102,104,106 \mathrm{Pd}$ turn out more or less as expected (fig. 9) (i.e. the $R_{6}$ and $R_{8}$ values agreed with VMI predictions (and for $98 \mathrm{Pd}$ and $102 \mathrm{Pd}$ the agreement continues to $\mathrm{J}=12$ and 14 , respectively)), the spectrum of $100 \mathrm{Pd}$ displays an increasing downward deviation from the VMI predictions, indicating "backbending" already above the 4+ state. As $100 \mathrm{Pd}$ possesses four proton holes and four neutrons away from the magic number 50, this result suggests that also two nucleon pairs can couple to two hole pairs to form a pseudomagic nucleus, but that in this case the reduction in energy 
spacing is not as abrupt as in the case of one nucleon pair being coupled to one hole pair: here the $6+$ and $8+$ states are far from degenerate, implying that the moment of inertia does not increase as radically as in nuclei with one particle pair - one hole pair.

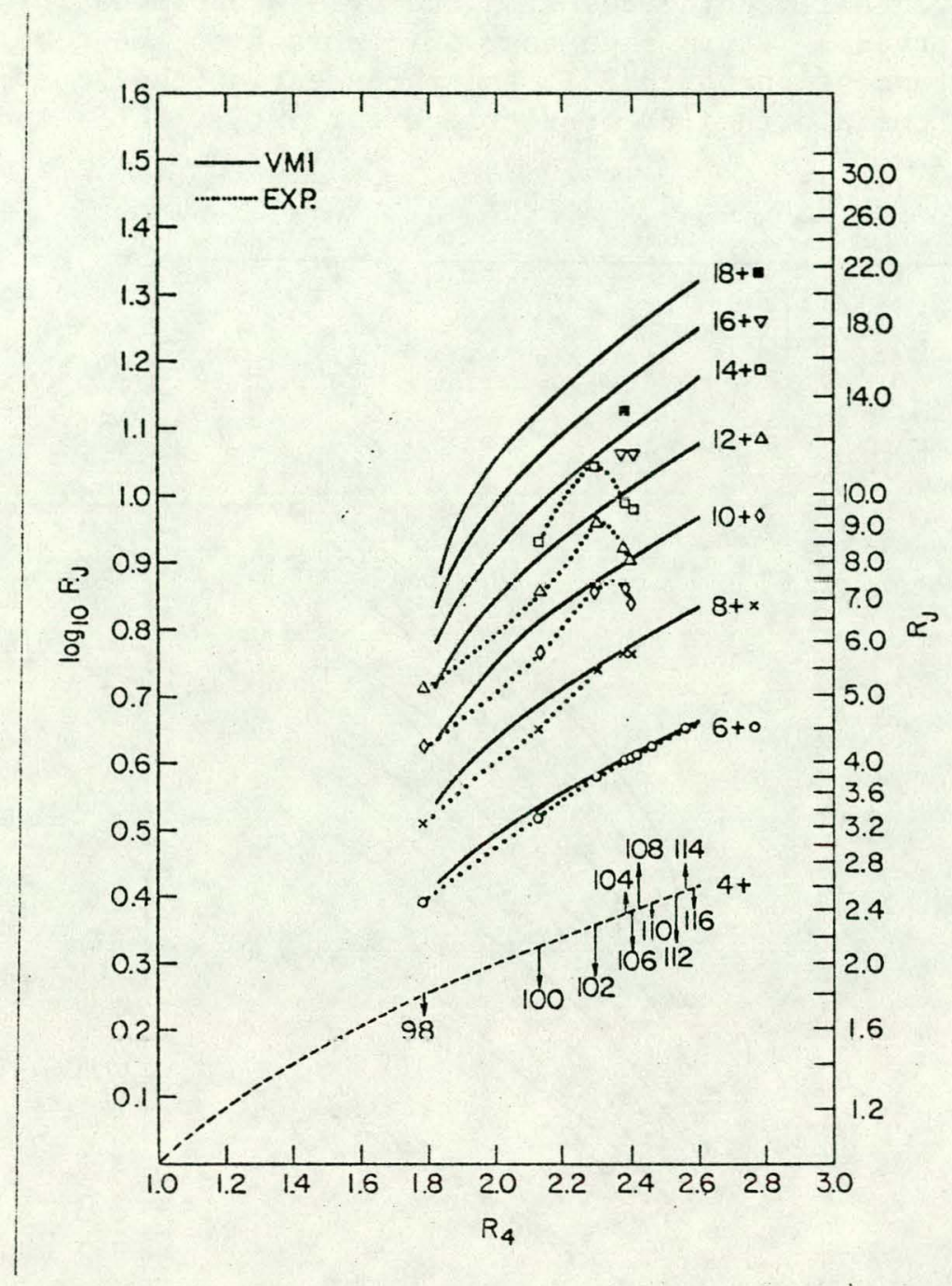

Fig. 9. Comparison of ground-state band energies in even-even Pd nuclei with predictions of the VMI model (full curves). The figure presents $\log R_{J}$ vs. $R_{4}$ (the lowest curve (short dashes) indicates $R_{4}$ on the logarithmic scale, with arrows pointing to the mass numbers of even-even Pd nuclei). It is seen that $R_{4}$ increases with increasing neutron number. In order to guide the eye, the experimental points are connected by dotted curves. The $R_{6}$ and $R_{8}$ values for $A \geq 102$ are seen to agree very well with the VMI predictions. The points for ${ }^{100} \mathrm{Pd}$ are somewhat depressed, while those for ${ }^{98} \mathrm{Pd}$, whose $\mathrm{R}_{4}(1.786)$ value lies slightly below the limit of validity (1.82) of the VMI model, are close to the continuation of the VMI curves. For $\mathrm{J} \geq 10$, only the values for $102 \mathrm{Pd}$ and $98 \mathrm{Pd}$ agree well with VMI, whereas the $\mathrm{R}_{J}$ values for $100 \mathrm{Pd}$ are increasingly depressed. We attribute this depression to the presence of four proton holes and four neutron particles, i.e. to the pseudomagic nature of this nucleus. 
Fig. 10 compares the experimental data also to the predictions from eq. 5. In order to carry out the comparison, we have extended the "Ejiri curves" to $\mathrm{R}_{4}=1.82$, the limit of validity of the VMI model. (One notices that below $\mathrm{R}_{4}=2$, the sequence of states according to the Ejiri formula becomes inverted, so that the $18+$ state eventually coincides with the $4+$ state. Behavior of this type has never been observed.) It is seen that in contrast to the good fit of the VMI model (with the exception of $100 \mathrm{Pd}$ ), the agreement of the Pd spectra with the Ejiri curves (which contain the IBM predictions for $\mathrm{SU}(3)$, $\mathrm{SU}(5)$ and $0(6)$ ) is far from satisfactory.

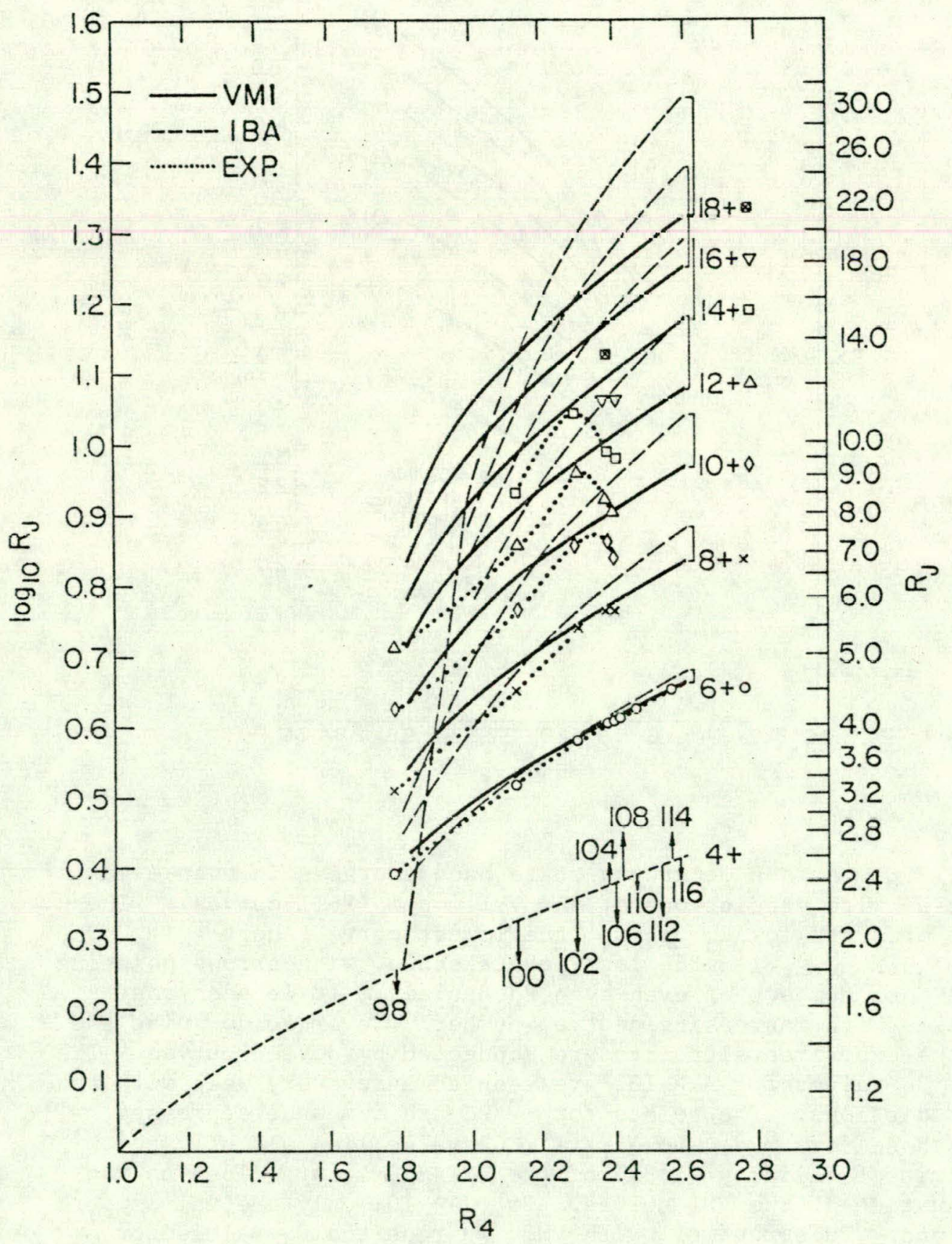

Fig. 10. This figure is identical to fig. 9 apart from the fact that experimental points are here also compared with the interacting boson approximation (IBA) or Ejiri predictions (eq. 5). It is noteworthy that the IBA (broken) curves tend to cross each other below $R_{2}=2$, i.e. the order of the predicted states in the band is reversed--a phenomenon which contradicts observation. The agreement of the $R_{6}$ and $R_{8}$ values with VMI is considerably better than with IBA. 
The hypothesis of the occurrence of pseudomagic structure can be further tested using all known spectra of nuclei possessing one (two) particle pairs - one (two) hole pairs, as shown in fig. 11. It is obvious that the expected features indeed occur in all cases known, namely, in ${ }_{18}^{40} \mathrm{Ar}_{22},{ }_{40}^{88} \mathrm{Zr}_{48},\left({ }^{88} \mathrm{Sr}\right.$ is considered to be doubly magic), ${ }_{22}^{48} \mathrm{Ti}_{26},{ }_{24}^{48} \mathrm{Cr}_{24},{ }_{54}^{132} \mathrm{Xe}_{78}$ and ${ }_{86}^{208} \mathrm{Rn}_{122}{ }^{16}$ ). The only exception is ${ }^{56} \mathrm{Fe}$, an isobar of ${ }^{56} \mathrm{Ni}$, whose $6+$ and $8+$ states are quite close to the VMI curves. It may be noted here that for the one particle pair - one hole pair excitation in ${ }^{48} \mathrm{Ti}$, level energies based on a generator coordinate method and on two different shell model calculations deviate appreciably from the experimental spectrum,

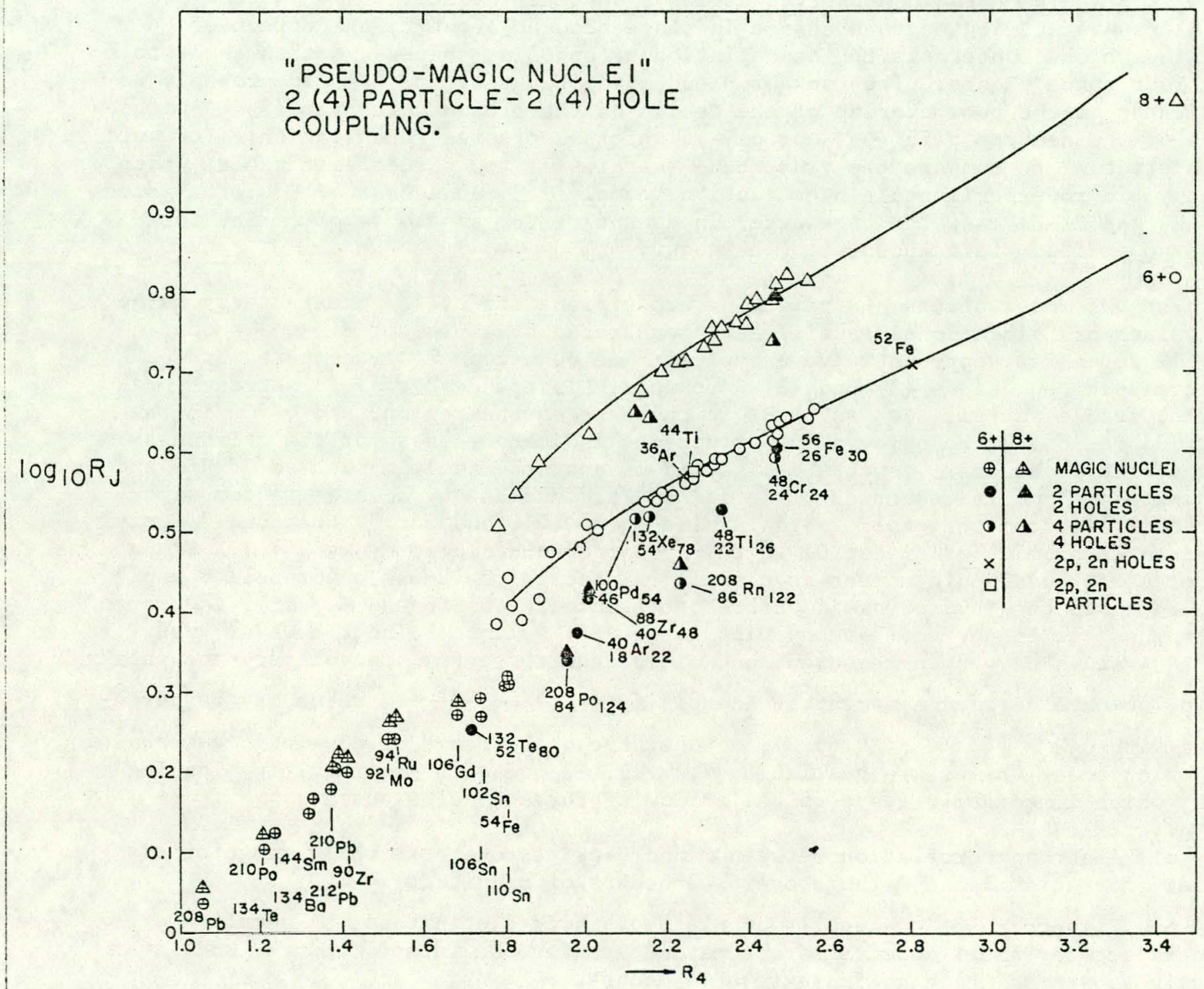

Fig. 11. The values $\log \mathrm{R}_{\mathrm{J}}$ for pseudomagic nuclei as well as for doubly and singly magic and "ordinary" non-magic nuclei are plotted against $\mathrm{R}_{4}$. (Recent energy values, taken from Sakai and Rester (1977), are used.) In one of the pseudomagic nuclei, namely ${ }^{132} \mathrm{Te}$, "backbending" occurs already above the $2+$ state, while in the remaining nuclei it occurs above the $4+$ state. In the nuclei containing two particles and two holes the transition is considerably more abrupt than in those containing four particles and four holes. Also shown are the $R_{6}$ values for nuclei with $2 \mathrm{p}, 2 \mathrm{n}$ holes ( $\mathrm{x})\left({ }^{36} \mathrm{Ar},{ }^{52} \mathrm{Fe}\right)$ and $2 \mathrm{p}, 2 \mathrm{n}$ particles ( $\left.\square\right)\left({ }^{44} \mathrm{Ti}\right)$, which coincide precisely with the VMI curve. (For the sake of clarity, the $R_{6}$ values for the neutron-rich ${ }^{124} \mathrm{Te}$ to ${ }^{130} \mathrm{Te}$ and Po nuclei are omitted from the graph.) 
although the two shell model calculations agree well with each other ${ }^{17}$ ). One may raise the question whether the presence of one valence proton pair and one valence neutron pair or of one proton hole pair and one neutron hole pair might also produce a pseudomagic spectrum. However, this is not the case. As is seen from fig. 11 , the $\mathrm{R}_{6}$ values for $38 \mathrm{Ar}_{18}(\mathrm{~h}-\mathrm{h}){ }_{22}^{4} \mathrm{Ti}_{22}(\mathrm{p}-\mathrm{p})$ and $52 \mathrm{Fe}_{26}(\mathrm{~h}-\mathrm{h})$ agree exce1lently with the VMI curve.

It seems plausible that the exceptional behavior of yrast bands in pseudomagic nuclei is related to the fact that the nuclear quadrupole moment changes sign at the magic number. Hence the presence of a particle pair and hole pair may be expected to bring about a partial cancellation of the prolate versus the oblate shape $\left.{ }^{18}\right)$. The backbending observed at low $\mathrm{J}$ in these nuclei $(\mathrm{J} \leq 4$ instead of $\mathrm{J} \geq 8$ ) may be attributed to a change in shape brought about by the effect of cranking which counteracts the cancellation of opposing shapes and thus leads to an abrupt shape change. The lack of pseudomagic behavior in ${ }^{56} \mathrm{Fe}$ can probably be attributed to the poor overlap of the orbits of the proton hole pair $\left(f_{7 / 2}\right)$ with those of the neutron pair $\left(\mathrm{p}_{3 / 2}\right.$ or $\left.\mathrm{p}_{1 / 2}\right)$, which suppresses cancellation. It would be interesting to compare the yrast band energies of two pseudomagic nuclei which might yield to experimental investigation, namely ${ }^{16} \mathrm{C}$, an isobar of ${ }^{16} \mathrm{O}$, for which only $E_{2}$ and $E_{4}$ have so far been determined, and $100 \mathrm{Cd}$, an isobar of $100 \mathrm{Sn}$, for which no excited state appears to be kuluwu.

3). The VMI model offers a straightforward explanation of the unexpectedly large $\mathrm{Q}_{2+}$ values of "vibrational" nuclei as demonstrated by a plot of $d \mathrm{vs}$. J shown in fig. 12 for a few representative examples. The functions $\checkmark(J)$, computed from the spectra using equations (1) and (3), are fairly independent of $\mathrm{J}$ for strongly deformed, stable nuclei, such as $180 \mathrm{Hf}$ in the rare earths region, and $248 \mathrm{Cm}$ in the actinide region. (The numbers in parentheses indicate values for the stiffness parameter C.) However, moments of inertia of unstable nuclei, such as $17 \mathrm{Hf}_{\mathrm{H}}$, and "transitional" nuclei, such as ${ }^{120} \mathrm{Xe}$ and ${ }^{194} \mathrm{Pt}$, increase by a large factor with increasing $\mathrm{J}$, from the small initial value $f_{0}=\mathcal{S}(0)$, and for "vibrational" nuclei Sincreases from 0 (or almost 0 ) to fairly large values already at $\mathrm{J}=2$. Since the electric quadrupole moment is closely related to the "mass quadrupole moment" (according to the "hydrodynamic" Bohr-Mottelson model $Q \propto \beta$ and $\mathcal{S} \propto \beta^{2}$ ), the large $Q_{2+}$ values are in perfect harmony with large $S(2)$ values. Since, however, very few $\mathrm{Q}_{2}+$ values have been measured, and since even these few are not very accurately known, we have defined a moment of inertia $g_{02}=\frac{g(0)+f(2)}{2}$, which may be correlated with $Q_{02}=[16 \pi / 5 B(E 2)(0 \rightarrow 2)]^{1 / 2}$ over the whole range of non-magic e-e nuclei for which these values are available $\left.{ }^{19}\right)$. Fig. 13 presents the resulting $\log \log$ plnt, which immediately leads to the following three conclusions:

a) A strong correlation between $\mathcal{S}$ and $Q$ exists over the whole range of nuclei (for which $\mathfrak{S}$ and $Q$ range over $\sim 2$ orders of magnitude).

b) This correlation clearly consists of three distinct parts, namely a linear part for small moments, a quadratic part for the larger moments, and finally a part in which no increase of $\mathbb{S}$ occurs.

c) No dependence on either $A$ or $Z$ is observed, whereas the B.M. "hydrodynamic prediction" gives $S / Q^{2} \propto A^{1 / 3} / Z^{2}\left(\approx A^{-5 / 3}\right)$.

The linear part consists of light, deformed nuclei such as ${ }^{12} \mathrm{C},{ }^{24} \mathrm{Mg}$, ${ }^{32} \mathrm{~S}$, etc., as well as of medium weight $(A \sim 100)$ and heavy (A $~ 200)$ "vibrational" nuclei, all characterized by possessing not more than 2 valence nucleon pairs away from one closed shell. The straight line drawn through the experimental values corresponds to the equation $\delta=\frac{m}{e} Q$, where $m$ denotes the nucleon mass and $e$ the unit charge. The absolute magnitude of $f$ in this region is described to an accuracy of $\pm 50 \%$ by a rotating Alpha-Dumbbell model with 


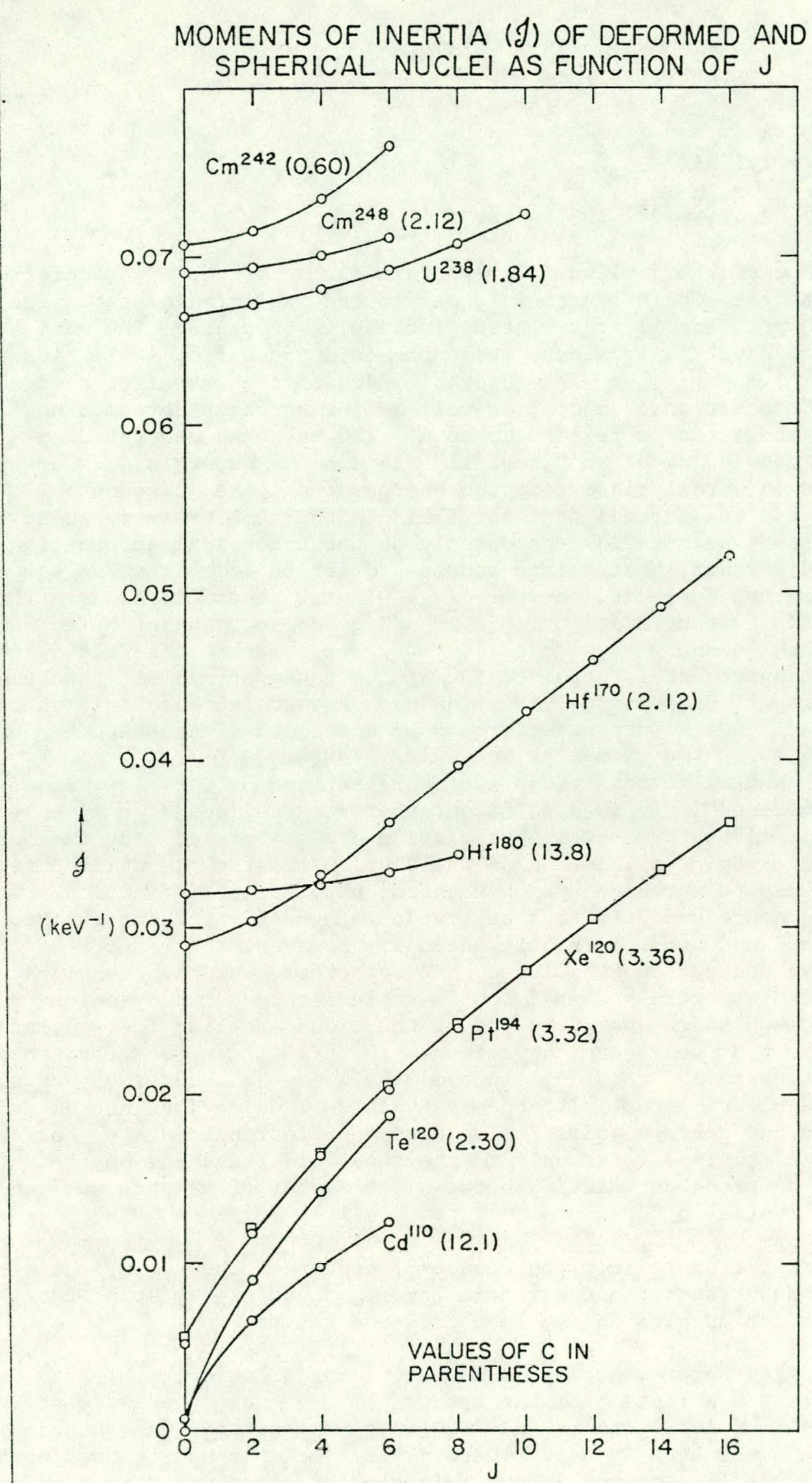

Fig. 12. Some representative examples of rotational bands (Hf ${ }^{180}$, $\left.\mathrm{Hf}^{170}, \mathrm{U}^{238}, \mathrm{Cm}^{242}, \mathrm{Cm}^{248}\right)$, bands in transition nuclei ( $\mathrm{Xe}^{120}$, $\mathrm{Pt}^{194}$ ' and "vibrational" bands ( $\left.\mathrm{Cd}^{110}, \mathrm{Te}^{120}\right)$ are shown. Values for the stiffness parameters $C$ in units of $10^{6} \mathrm{keV}^{3}$ are given in parentheses. This figure was first published in ref. 8 . 


$$
\begin{aligned}
& g=2 \mathrm{M}_{\alpha} \mathrm{R}^{2} \approx 2.4 \times 10^{-4} \mathrm{~A}^{2 / 3} \mathrm{keV}^{-1} \\
& Q=4 \mathrm{e}_{\alpha} \mathrm{R}^{2} \approx 9.6 \times 10^{-2} \mathrm{~A}^{2 / 3} \mathrm{eb} \\
& \mathrm{R}-1.1 \Lambda^{1 / 3} \mathrm{fm}
\end{aligned}
$$$$
\Im / Q \approx 2.41 \times 10^{-3}(\mathrm{eb})^{-1} \mathrm{keV}^{-1}
$$

where $R$ is the radius of the nucleus. The picture is one of two $\alpha$ particles connected by a massless rod, whose length is equal to the nuclear diameter. The departures of $\mathcal{S}$ and $Q$ are strongly correlated with shell structure: The most nearly magic nuclei tend to have small values, while those furthest from shell closure have values up to $\approx 1.5 \times \oiint_{\text {model }}$. The quadratic part of the correlation which includes values for "transitional" nuclei as well as for strongly deformed nuclei in the rare earths and actinide regions up to $A \sim 230$ has been described by a "two-fluid" model, consisting of an "inertial" fluid and a superfluid, whereby the former participates in normal rigid rotation whereas the latter does not. It is assumed that the ratio of inertial to total fluid is the same at every point of a given nucleus, and that this ratio depends only on the spheroldal deformaliun deduced from $Q_{n}$; and further, that charge and mass distributions are homogeneous. Fig. 14 compares the model predictions for $\$$ vs. $Q^{2}$ with observed values. Whe two-fluid model leads to the relation $\$ / Q^{2} \propto A^{5 / 3} / Z^{2} \approx$ const., where the approximate constancy follows because above mass $100, \mathrm{Z}$ rises somewhat more slowly than A. While excellent agrecment is obtained for a wide range of nuclei, the heaviest nuclei, which are all spontaneously fissioning, show no increase in $\checkmark$ whereas $Q$ values increase by $\sim 50 \%$. This behavior may be due to the fact that these nuclei may have a slight proton excess at the poles brought about by

Coulomb repulsion. Nuclei in this group are characterized by very low values for the stiffness parameters $C$. It is significant that for nuclides of a given species decreasing half-lives for spontaneous fission are associated with decreasing $\left(\mathrm{v}\right.$ values $\left.{ }^{20}\right)$. A microscopic explanation of the $\mathbb{f} \mathrm{vs}$. Q relation is still outstanding, although one was attempted ${ }^{21}$ ) by a new approach to collective motion which leads naturally and nonredundantly to a separation of the kinetic energy into rotational, vibrational and other intrinsic motions. However, the effects of the Pauli principle have not yet been included. Nevertheless, our phenomenological study of the moment of inertia has shed considerable light on the dynamics of nuclei with $\leq 2$ nucleon pairs away from one of the closed shells, i.e. nuclei corresponding to the B.M. weak coupling region: the transition from the twofluid type of rotation to the rotating alpha dumbbell may be thought of as a phase transition of the nuclear matter. It appears that IBM I which does not discriminate between proton and neutron pairs, as well as the "intrinsic state" corresponding to the geometric model ${ }^{22}$ ) refer only to the two-fluid phase (as may be gathered from the fact that a strong correlation between the number of valence nucleon pairs $\mathrm{N}$ and the basic VMI variable $R_{4}$ exists only for $\mathrm{N} \gtrsim 11$ ).

An interesting contribution to this conference ${ }^{23}$ ) concerned the comparison of moments of inertia and transition quadrupole moments up to higher spin states of yrast bands, in particular also in the band crossing region.

4.) The cubic equation for the nuclear moment of inertia, which, as we have seen, gives a rather precise description of the spectra of e-e nuclei near the groundstate, has two singularities: one at $\xi_{0}=0$, (which appears to be a second order phase transition) and one at $k_{0}=-\infty$, (where a first order phase transition takes $\mathrm{place})$. As we have seen in section 3 , the second order phase transition coincides exactly with the transition from the alpha-dumbbell configuration (or phase) to the two-fluid configuration. It is tempting, therefore, to compare this "A-body system" to many-body systems which are also, in first approximation, describable by cubic equations, such as the "van der Waals gas." I have compared these two systems a number of years $\mathrm{ago}^{24}$ ) on an intuitive basis, and concluded that the moment of inertia in the nuclear case appears to correspond to the density of 
the condensed gas, the angular momentum to the pressure, and the parameter $\mathcal{S}_{0}$ (or the number of valence nucleons) to the temperature.

Recently, in collaboration with Max Dresden who has become an expert on Catastrophe Theory, we have compared ${ }^{25}$ ) the VMI model for the nucleus with two systems of condensed matter, namely, in addition to the van der Waals gas, with a type of ferroelectric system called perovskite. (Catastrophe Theory is analogous to the LandauGinsburg theory of phase transitions; both approaches omit a treatment of critical

fluctuations.) Without giving a detailed account of this comparison, I should like to state here our main results: each of the three systems has a critical point (second order phase transition) when the two counteracting forces controlling it are in balance: In the nuclear case, the competition refers to the tendency to preserve the spherical shape of the closed shell which conflicts with the tendency to deform the nuclear shape; the parameter $\mathscr{S}_{0}$ vanishes when both tendencies are exactly in balance. In the van der Waals case these tendencies are just the repulsive and attractive regions of the intermolecular forces. In the ferroelectric case, the combating tendencies are the polarization which tends to pull the crystal apart, and the elastic forces tending to restore the original shape. Balance is established in this case when the dielectric constant $\varepsilon_{0}$ passes through infinity. A first order phase transition occurs in the nucleus, when $\Im_{0} \rightarrow-\infty$. We have discussed before the physical meaning of this first order phase transition which occurs when the closed shell is reached. In the van der Waals case, the corresponding situation occurs when the attractive force vanishes, and therefore, the critical temperature $\mathrm{T}_{c}=0$, so that it would take infinite pressure to liquefy the gas. In the ferroelectric case, the corresponding situation occurs when the dielectric constant $\varepsilon_{0} \rightarrow 0$, so that no externally imposed field can produce an internal electric field. In addition, we discussed the condensed matter analogies to the critical angular momentum state in the ground state band of the nucleus, at which a deviation from the VMI model occurs. The relationships between the variables and parameters in the three systems are deduced. Although these are somewhat more cumplex Lhan those "guessed" in ref. ${ }^{24}$ ), they are in general agreement with the latter.
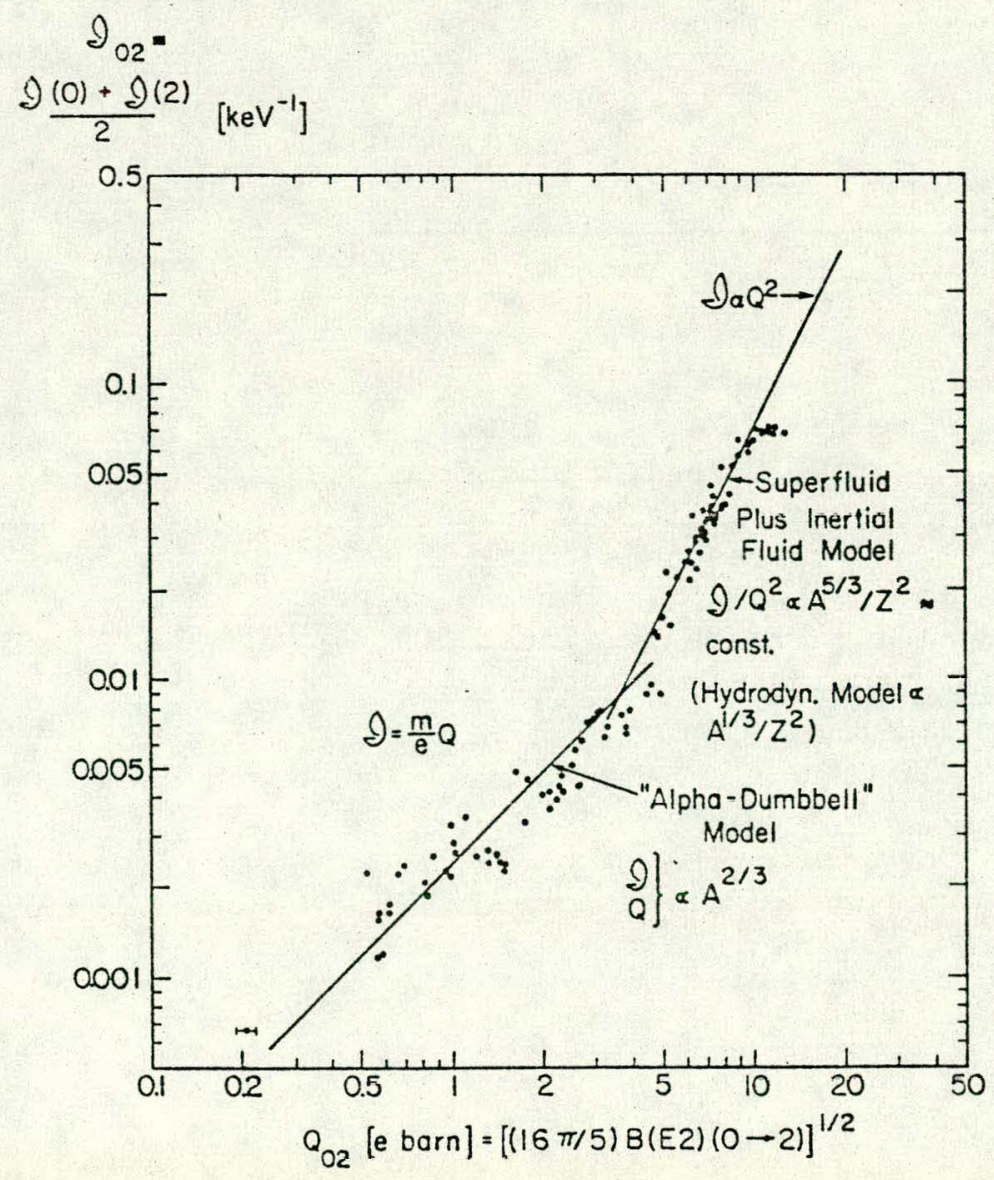

Fig. 13. Log $\log$ plot of the average moment of inertia $S_{02}=\frac{g(0)+S(2)}{2}$ vs. the transition quadrupole moment Q02. A linear part and a quadratic part can be clearly distinguished. The horizontal part for the highest \& $Q$ values refers to spontaneously fissioning actinides. The linear part is interpreted by the alpha-particle dumbbell model, the quadratic part by a macroscopic twofluid model. 


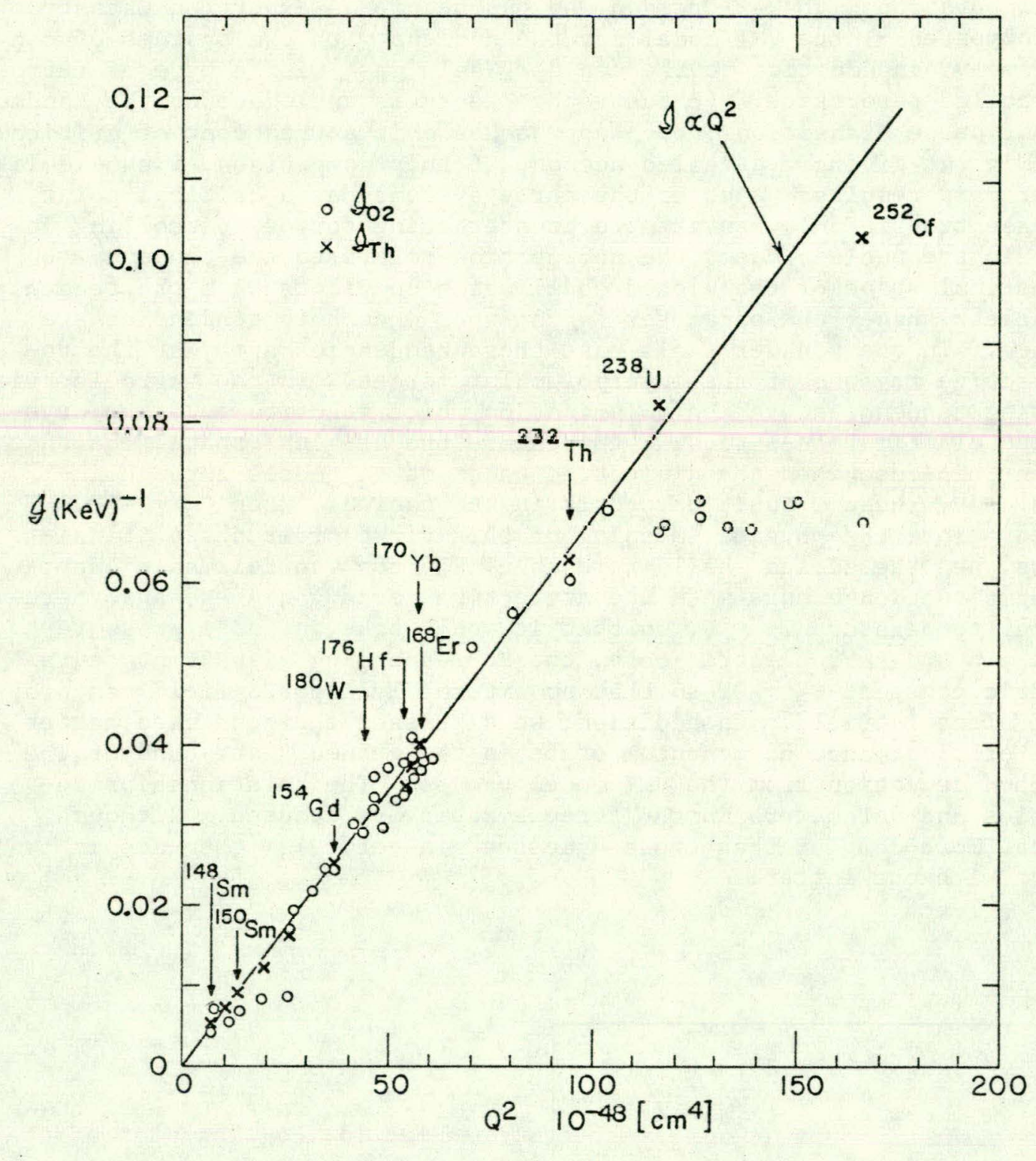

Fig. 14. Linear plot of $\Im_{02}$ vs. $Q_{02}^{2}$. Predictions from the two-fluid model are shown by crosses, empirical values by open circles. The straight line corresponds to $S_{02}=1 / \mathrm{k}^{2} \mathrm{Q}_{02}^{2}$, where $k=(39.4 \pm 2.6) \times 10^{-24} \mathrm{~cm}^{2}(\mathrm{keV})^{1 / 2}$. The $Q_{02}$ values for the heaviest actinide nuclei increase by almost $50 \%$, while the $\mathfrak{S}_{02}$ values remain approximately constant. 
It is a pleasure to express my warm gratitude to the many associates who were essential in helping, step by step, to reveal secrets so closely guarded by Nature. D. E. Alburger, Garman Harbottle, Guy Emery, W. R. Kane and M. L. Perlman deserve my thanks for their help with the pre-VMI phases of the experimental work. Of great value during that period was the knowledge of experimental results and the analyses arrived at--and freely communicated--by F. S. Stephens and R. M. Diamond. During the early VMI phase the great enthusiasm and ingenuity of Mario Mariscotti, together with the insights contributed by Brian Buck were essential. Since then the depth of theoretical knowledge, inspired cooperation, and ever-ready sage advice of Fred Goldhaber proved to be invaluable. The experimental help needed to test various aspects of the VMI model was generously given by S. Cochavi, 0. C. Kistner, A. H. Lumpkin and especially W. F. Piel, Jr. (who continues to be associated with this work up to the present time). Of particular importance for more than twenty years was the resourceful, intelligent, and joyful assistance, both in the experimental and analytical aspects of the work, by Michael McKeown. For the theoretical sections of the review ${ }^{2}$ ) I could not have found more thoughtful and knowledgeable associates than Carl B. Dover and Alan L. Goodman. For work on the still somewhat open question of the applicability of the VMI model to reaction theory I am indebted to A. Arima and K. McVoy. I also enjoyed the collaboration with J. D. Garrett and J. P. Vary on the role of the stiffness parameters of spontaneously fissioning actinides. J. Rasmussen and his associates made valuable contributions to the application of the VMI model to the spectra of odd A nuclei and to the elucidation of the relation of the phenomenological VMI parameters to microscopic theory. A. Faessler and his associates have been for many years intrigued by the phenomena described above and have made valuable contributions to their theoretical analysis, especially with regard to the spectra of triaxial nuclei, and of the behavior of higher lying bands. S. M. Harris has long been a valuable judge of controversial theoretical approaches. Further, I wish to thank L. Zamick fur his longstanding interest and his contribution to the understanding of pseudomagic nuclei, and to M. Dresden for his help in exploring analogies between laws describing the behavior of condensed matter and the VMI equations. Finally, I wish to pay tribute to the attention given to this work by A. Bohr and B. R. Mottelson, as well as by A. Klein and L. C. Biedenharn, although (or possibly even because) each one of them played at times an adversary role.

\section{REFERENCES}

"Research supported by the U. S. Department of Energy under Contract Nos. DE-AC02-80ER10576 and DE-ACO2-76CH00016.

1) P. C. Simms, F. A. Rickey and R. K. Popli, invited paper to this conference.

2) G. Scharff-Goldhaber, C. Dover and A. L. Goodman, Ann. Rev. Nuc1. Sci 26 (1976) 239 and references therein.

3) C. Mallmann, Phys. Rev. Lett. 2 (1959) 507.

4) The fact that a deforming (quadrupole-quadrupole) interaction takes place only between pairs of unlike particles, was recently again emphasized by I. Talmi in Neutron capture gamma-ray spectroscopy, ed. R. E. Chrien and W. R. Kane (Plenum Press, New York and London, 1978) p. 1.

5) G. Scharff-Goldhaber, Proc Pittsburgh Conference, 1957, ed. S. Meshkov, p. 447.

6) G. T. Emery, W. R. Kane, M. Mckeown, M. L. Perlman and G. Scharff-Goldhaber, Phys. Rev. 129 (1963) 2597.

7) M. A. J. Mariscotti, G. Scharff-Goldhaber and B. Buck, Phys. Rev. 178 (1969) 1864.

8) G. Scharff-Goldhaber and A. S. Goldhaber, Phys. Rev. Lett. 24 (1970) 1349.

9) T. K. Das, R. M. Dreizler and A. Klein, Phys. Rev. C 2 (1970) 632.

10) G. Scharff-Goldhaber, J. Phys. G: Nuc1. Phys. 5 (1979) L207; Corrigenda 6 (1980), No. 3 . 
11) G. Scharff-Goldhaber, J. Phys. A: Math. Nuc1. Gen. 7 (1974) L212.

12) S. Cochavi, O. Kistner, M. Mckeown and G. Scharff-Goldhaber, J. Phys. France 33 (1972) 102.

13) G. Scharff-Goldhaber, M. WcKeown, A. H. Lumpkin and W. F. Mie1, Jr., Phys. Lett. B 44 (1973) 416.

14) W. F. Piel, Jr., G. Scharff-Goldhaber, A. H. Lumpkin, Y. K. Lee and D. C. Stromswold, to be published.

15) W. F. Piel, Jr., and G. Scharff-Goldhaber, Bul1. Am. Phys. Soc. 23 (1978) 555.

16) H. Backe, et al., Ann. Rep., MPI, Heidelberg, (1977) 121; H. K. Carter, private communication.

17). M. Faber, A. Faessler and H. Muether, Z. Physik A 285 (1978) 77. Other attempts at shell model calculations of the $48 \mathrm{Ti}$ spectrum have similar flaws (G. Fortuna, et a1., Nuov. Cim. 34A (1976) 321; B. J. Linard, et a1., Nuc1. Phys. A302 (1978) 214).

18) I am indebted to L. Zamick for this suggestion.

19) A. S. Goldhaber and G. Scharff-Goldhaber, Phys. Rev. C 17 (19/8) 1171.

20) J. D. Garrett, G. Scharff-Goldhaber and J. P. Vary, Bull. Am. Phys. Soc. 19 (1974) 59.

21) B. Buck, L. C. Biedenharn and R. Y. Cusson, Nuc1. Phys. A317 (1979) 205.

22) J. N. Ginocchio and M. W. Kirson, Proc. Int. Conf. on band structure and nuclear dynamics, New Orleans, 1980, ed. A. L. Goodman, Vo1. 1, contributed papers, p. 38 .

23) W. Andrejtscheff, E. Nadjakov and Ts. Venkova, Proc. Int. Conf. on band structure and nuclear dynamics, New Orleans, 1980, ed. A. L. Goodman, Vo1. 1, contributed papers, p. 17.

24) G. Scharff-Goldhaber in Atomic masses and fundamental constants 4 (Plenum Press, New York, 1972)p. 272.

25) G. Scharff-Goldhaber and M. Dresden, Application of catastrophe theory to nuclear structure, in: Festschrift for M. Goldhaber (to be published by New York Academy of Sciences), BNL-27077. 